\title{
Correlation between apparent diffusion coefficient and Ki-67 in different pathological types of lung cancer
}

\author{
Hongna Ren, Jianbing Ma, Jia Wang \\ Department of Radiology, the First Hospital of Jiaxing, The Affiliated Hospital of Jiaxing University, Jiaxing, China \\ Contributions: (I) Conception and design: J Ma; (II) Administrative support: J Ma; (III) Provision of study materials or patients: J Ma; (IV) Collection \\ and assembly of data: H Ren; (V) Data analysis and interpretation: H Ren; (VI) Manuscript writing: All authors; (VII) Final approval of manuscript: \\ All authors. \\ Correspondence to: Jianbing Ma. Department of Radiology, the First Hospital of Jiaxing, The Affiliated Hospital of Jiaxing University, 1882 South \\ Zhonghuan Road, Jiaxing 314000, China. Email: 413947372@qq.com.
}

\begin{abstract}
Background: Computed tomography (CT) is common and valuable non-invasive method to evaluate proliferative activity in lung cancer; however, magnetic resonance (MR) is rarely used in lung cancer. To evaluate whether there were different expression levels of Ki-67 in different pathological types of lung cancer and the correlation between Ki-67 index and apparent diffusion coefficient (ADC) value.

Methods: The clinical and pathological data of 165 patients with lung cancer confirmed by needle biopsy or surgery were retrospectively evaluated, among whom 54 underwent MR examination. The level of Ki-67 was tested using the international standard IHC 2-step method. Kruskal-Wallis H test was used to compare the different expression level of Ki-67 index of adenocarcinoma, squamous cell carcinoma, and small cell carcinoma, and the least significant difference (LSD) method was used for pair-group comparison. Spearman correlation coefficient was used to analyze the correlation between Ki-67 index and ADC value. All statistical tests were bilateral, and $\mathrm{P}<0.05$ was considered statistically significant.

Results: The median of Ki-67 index in adenocarcinoma, squamous cell carcinoma, and small cell carcinoma were $20(10,30), 40(40,60)$, and $70(60,80)$, respectively. There was a statistical difference in the expression level of Ki-67 index in the 3 different pathological types of lung cancer $(\mathrm{H}=103.762, \mathrm{P}=0.000)$. In 54 cases of lung cancer with MR examination, there was a significant negative correlation between average ADC value and expression level of Ki-67 index ( $\mathrm{r}=-0.506, \mathrm{P}=0.000)$, while there was no correlation between ADC value and Ki-67 index in distinguishing different pathological types of lung cancer.

Conclusions: The expression level of Ki-67 index was different among different pathological types of lung cancer. The ADC value is correlated with $\mathrm{Ki}-67$ index in lung cancer, which can be used to evaluate the proliferative activity of lung cancer.
\end{abstract}

Keywords: Lung cancer; magnetic resonance (MR); Ki-67 index; diffusion weighted imaging (DWI)

Submitted Oct 21, 2021. Accepted for publication Dec 08, 2021.

doi: $10.21037 /$ tcr-21-2515

View this article at: https://dx.doi.org/10.21037/tcr-21-2515

\section{Introduction}

Lung cancer is one of the most common malignant tumors in the world (1), and is associated with a low 5 -year survival rate. The most important prognostic factor of lung cancer is local and distant metastasis. The nuclear protein, Ki67 , is an important index reflecting the proliferation status in lung cancer cells (2), and the Ki-67 proliferation index
(PI) has been widely used as a marker of cell proliferation. Previous studies have shown that a high Ki-67 PI has a negative impact on disease-free survival and relapse-free survival $(3,4)$. Although Ki-67 index can adequately reflect the metastasis and prognosis in lung cancer, the proliferative activity of lung cancer often needs to be evaluated by invasive surgery or biopsy. Currently, there are many 
imaging techniques for the diagnosis of lung cancer, such as X-ray, computed tomography (CT), positron emission tomography (PET) and magnetic resonance $(\mathrm{MR})(5,6)$. Among them, diffusion weighted imaging (DWI) sequence of MR has been reported as useful for the differential diagnosis of malignant and benign tumors in the lung (7-10). In addition, 2 meta-analyses have also shown that DWI is useful for the evaluation of the node $(\mathrm{N})$ factor of lung cancer $(11,12)$. MR has higher sensitivity and accuracy in distinguishing the solid components or necrotic cystic areas of lung cancer than CT examination. Compared with CT, MR can provide more information about the components of the lesion by multi-sequence imaging, and is safer without radiation. Therefore, MR has been increasingly applied in the research of lung cancer in recent years.In this study, the correlation between Ki-67 index and apparent diffusion coefficient (ADC) value was analyzed to evaluate its feasibility and reliability in predicting the proliferative activity of lung cancer. In our study, a relatively large number of patients were included to verify the expression level of Ki-67 index in different pathological types of lung cancer. Comparing the correlation between $\mathrm{Ki}-67$ index and ADC value, a relatively large number of patients were also enrolled for the MR examination, and there were relatively equal numbers of patients between the different groups in order to reduce the error. In addition, $\mathrm{Ki}-67$ index was compared in the different pathological types of lung cancer.

We present the following article in accordance with the MDAR reporting checklist (available at https://dx.doi. org/10.21037/tcr-21-2515).

\section{Methods}

This study was approved by the Ethics Committee of the First Hospital of Jiaxing (No.: LS2018-221), and the requirement for individual consent was waived by the committee due to retrospective nature of the study. All procedures performed in this study involving human participants were in accordance with the Declaration of Helsinki (as revised in 2013).

\section{Patients}

From March 2012 to June 2021, 165 patients with pathologically confirmed lung cancer (140 men and 25 women; age range, 44 to 88 years; mean age, 70 years) were recruited to this study. Pathological assessment revealed 47 adenocarcinomas, 58 squamous cell carcinomas, and 60 small cell carcinomas. None of the patients had received chemotherapy or radiotherapy prior to confirmation of their diagnosis.

There were 57 patients who underwent MR examination. Among them, 3 patients were excluded owing to having received chemotherapy or targeted drugs before MR examination. Finally, 54 patients with a single solid tumor were included. The workflow of patient selection is shown in Figure 1.

The inclusion criteria were as follows: (I) patients with lung cancer confirmed by needle biopsy or surgery; (II) complete MR imaging data; (III) well-defined measurable areas of tumor tissue were displayed on MR images; (IV) immunohistochemistry was performed, and Ki-67 index was positive.

The exclusion criteria were as follows: (I) MR examination had not been performed in our hospital; (II) the ADC value could not be measured on MR images; (III) had already received chemotherapy or other treatments before MR examination.

\section{Image acquisition}

All MR images were obtained by 3.0-T unit (GE Discovery MR750; GE Healthcare, Waukesha, WI, USA) equipped with an 8-channel body coil.

A supine position was adopted and breathing exercises were carried out before scan. The scanning sequences included T1WI [repetition time (TR) 600-900 ms, time to echo (TE) $5.8 \mathrm{~ms}$, layer thickness $6.0 \mathrm{~mm}$, layer spacing $1.2 \mathrm{~mm}$, horizontal axis], T2WI (TR 6,000-8,000 ms, TE $80 \mathrm{~ms}$, layer thickness $6.0 \mathrm{~mm}$, layer spacing $1.2 \mathrm{~mm}$ ), and DWI (respiratory navigation single excitation plane echo imaging; B value was $0,100,200,500,600,800$, and $1,000 \mathrm{~s} / \mathrm{mm}$, respectively). Ultimately, the $B$ value of $500 \mathrm{~s} / \mathrm{mm}$ was selected for image analysis.

\section{Image analysis}

On the ADW 4.2 post-processing workstation (GE Healthcare, USA), the Function Tool software was used for image post-processing to evaluate DWI signal intensity characteristics and measure ADC value. The region of interest (ROI) selection was as follows: the area with the largest and most uniform signal intensity of the lesion was selected for measurement (signal intensity was taken the mean value of 3 measurements). For the more homogeneous 


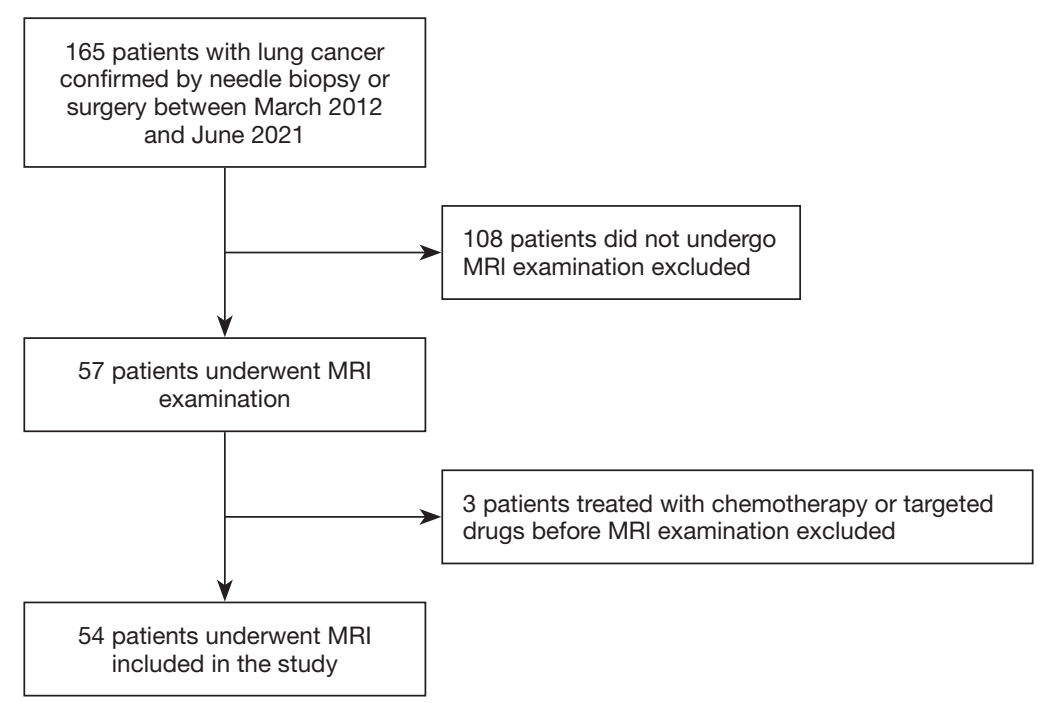

Figure 1 Workflow of patient selection.

lesions, the ROI included more than $50 \%$ of the maximum diameter of the lesion. For the inhomogeneous lesions with cavities, liquefying, or necrotic components, multiple smaller areas of ROI were selected to calculate the mean value (Figure 2), including the center area of the maximum signal intensity as far as possible. The range of ROI was $20-500 \mathrm{~mm}^{2}$. The data were analyzed by 3 radiologists experienced in chest MR imaging diagnosis using a doubleblind method, and the mean value was obtained.

\section{Pathologic reviews}

The expression level of Ki-67 was routinely evaluated by 2 pathologists (with 14 and 15 years of experience in phymatology, respectively) using typical tumor samples collected from the patients. Routine hematoxylin and eosin (HE) and immunohistochemical (IHC) staining were performed and recorded. The level of Ki-67 was tested using the international standard IHC 2-step method, and defined as positive when the nucleus was stained brown. The Ki-67 index was expressed using the percentage (\%) of positive cells under low-to high-power microscopy.

\section{Statistical analysis}

Statistical analysis was carried out using SPSS 26.0 software (IBM Corp., Armonk, NY, USA). For quantitative variables, those with normal distribution were represented by $\bar{x}_{ \pm \mathrm{S}}$, and those with non-normal distribution were represented by median (P25, P75). Kruskal-Wallis H test was used to compare the expression level of Ki-67 index of adenocarcinoma, squamous cell carcinoma, and small cell carcinoma. The variables were ranked for pairwise comparison between groups, and the rank was used as the measurement variable. The least significant difference (LSD) method was used for pair-group comparison. The relationship between ADC value and $\mathrm{Ki}-67$ index was calculated by Spearman's coefficient. The difference was considered statistically significant when $\mathrm{P}<0.05$.

\section{Results}

A total of 165 patients with single lesions of lung cancer were included in our study. The clinical characteristics are summarized in Table 1. The median age was 70 years (range: 44 to 88 years) and the majority of patients were male (85\%). Among the types of tumors, 47 were adenocarcinomas (27\%), 58 were squamous cell carcinomas $(35 \%)$, and 60 were small cell carcinomas (36\%). The average Ki-67 index was $54.8 \%$ (range: $5 \%$ to $90 \%$ ).

A total of 54 patients underwent MR examination, among whom there were 17 adenocarcinomas (31\%), 17 squamous cell carcinomas (31\%), and 20 small cell carcinoma (38\%). The mean tumor diameter was $37.7 \mathrm{~mm}$ (range: 22.1 to $74.5 \mathrm{~mm}$ ).

The different expression level of $\mathrm{Ki}-67$ in the 3 pathological types of lung cancer are shown in Table 2 and Figure $3(\mathrm{H}=103.762, \mathrm{P}=0.000)$. Small cell carcinoma had the highest expression level and adenocarcinoma had the lowest. 

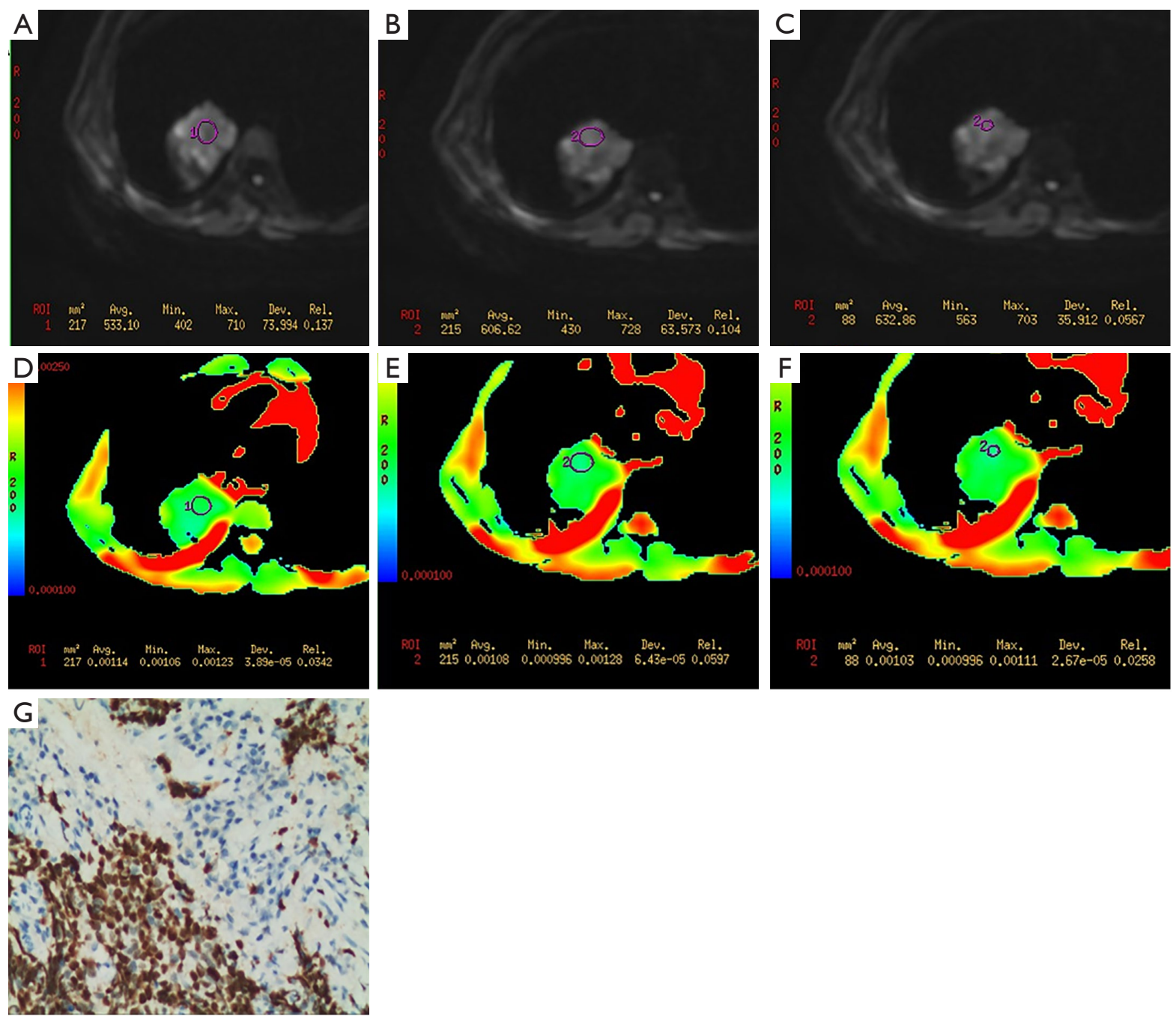

Figure 2 Small cell carcinomas in a 75 -year-old man who underwent MR examination. Comparing the DWI image (A-C, circle and numbers), three areas of ROI were selected on ADC map (D-F, circle and numbers), and the area of cavities, liquefying, or necrotic components were avoided. The area range of ROI was 88 to $217 \mathrm{~mm}^{2}$ (A-F circle and numbers). Immunohistochemistry map showed the high Ki-67expression (original magnification, ×200) (Ki-67=90\%) (G). ROI, region of interest; ADC, apparent diffusion coefficient; DWI, diffusion weighted imaging.

The correlation detected between $\mathrm{Ki}-67$ index and ADC value of lung cancer is summarized in Table 3. The average ADC value of the 3 types lung cancer was $(1.183 \pm 0.466) \times 10^{-6} \mathrm{~mm}^{2} / \mathrm{s}$. The average ADC values of adenocarcinoma, squamous cell carcinoma, and small cell carcinoma were $(1.299 \pm 0.451) \times 10^{-6} \mathrm{~mm}^{2} / \mathrm{s}$, $(1.232 \pm 0.437) \times 10^{-6} \mathrm{~mm}^{2} / \mathrm{s}$, and $(1.042 \pm 0.317) \times 10^{-6} \mathrm{~mm}^{2} / \mathrm{s}$, respectively. The average ADC value of the 3 types of lung cancer was negatively correlated with Ki-67 index ( $r=-0.506$, $\mathrm{P}=0.000$ ). The $\mathrm{ADC}$ value correlated moderately with Ki67 index (Figure 4). There was no correlation between ADC value and $\mathrm{Ki}-67$ index in the case of distinguishing the pathological types.

\section{Discussion}

The nuclear protein $\mathrm{Ki}-67$ is a monoclonal antibody located in the nucleus, which is related to cell mitosis and cycle, and can reflect the proliferation ability of tumor cells. The expression level of Ki-67 is different in benign and malignant tumors. Similarly, it is different in the pathological subtype of lung cancer. As the expression 
level of Ki-67 index increases, the proliferative activity of lung cancer increases, and the prognosis and survival rate decrease (5,9-11). The DWI technology can be used for the measurement and imaging of water molecular diffusion in vivo in a non-invasive form, and indirectly reflect the information of tissue structure and cell density at a micro molecular level. ADC value is used to represent the diffusion velocity and range of different directional water of DWI, which are mostly represented by ADC diagrams and values. ADC value mainly reflects the diffusion amplitude and signal level of water molecules, while is opposite to

Table 1 Clinical characteristics of patients with lung cancer

\begin{tabular}{lcc}
\hline Parameters & $\begin{array}{c}\text { Number of patients } \\
(\mathrm{n}=165)\end{array}$ & $\%$ \\
\hline Age (years) & 70 & \\
Average & $44-88$ & \\
Range & & 85 \\
Gender & 140 & 15 \\
Male & 25 & \\
Female & & 27 \\
Type & 47 & 35 \\
Adenocarcinoma & 58 & 36 \\
Squamous cell carcinoma & 60 & \\
Small cell carcinoma & & \\
Ki-67 index (\%) & 54.8 & \\
Average & $5-90$ & \\
Range & &
\end{tabular}

DWI. By quantifying the movement of water molecules, ADC value can indirectly reflect tumor cell density, peripheral invasion status and nucleo-plasma ratio to some extent. Due to the small extracellular space of tumor tissues, the diffusion of water molecules is limited, resulting in low $\mathrm{ADC}$ value. In terms of the choice of $\mathrm{B}$ value, previous studies have suggested that when the B value is $500 \mathrm{~s} / \mathrm{mm}$, it is less affected by other factors such as perfusion $(7,12,13)$. So, this value was also used in this study.

In this study, there were differences in the expression level of Ki-67 index among different pathological types of lung cancer, the average value of Ki-67 index in smallcell carcinoma was the highest, and it was the lowest in adenocarcinoma. This suggested that the division rate and count of tumor cells were different in different

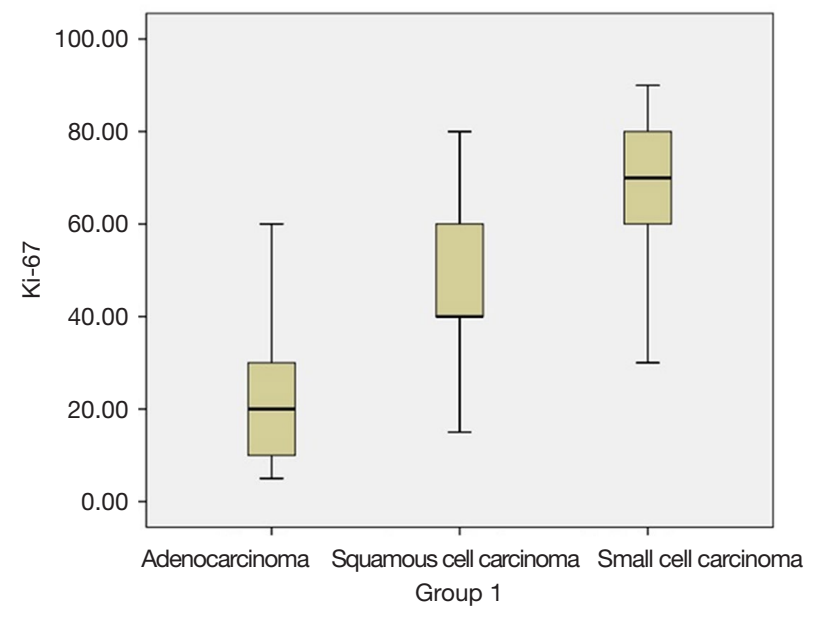

Figure 3 Distribution of Ki-67 index of 3 groups of lung cancer ( $\mathrm{H}=103.762, \mathrm{P}=0.000)$.

Table 2 Comparison of Ki-67 index among 3 groups of lung cancer

\begin{tabular}{lcc}
\hline Group & Number of patients & Ki-67 index (\%) \\
\hline Adenocarcinoma & 47 & $20(10,30)$ \\
Squamous cell carcinoma & 58 & $40(40,60)$ \\
Small cell carcinoma & 60 & $70(60,80)$ \\
Overall comparison & H=103.762 & $\mathrm{P}=0.000$ \\
Post Hoc (LSD, P) & Adenocarcinoma vs. squamous cell carcinoma & $\mathrm{P}=0.000$ \\
& Adenocarcinoma vs. small cell carcinoma & $\mathrm{P}=0.000$ \\
& Squamous cell carcinoma vs. small cell carcinoma & $\mathrm{P}=0.000$ \\
\hline
\end{tabular}

LSD, least significant difference. 
Table 3 Correlation between Ki-67 index and ADC value in lung cancer

\begin{tabular}{lcccc}
\hline & & \multicolumn{3}{c}{ Ki-67 index } \\
\cline { 2 - 5 } Parameter (ADC value) & Average & Adenocarcinoma & Squamous cell carcinoma & Small cell carcinoma \\
\hline$r$ & -0.506 & -0.294 & -0.375 & -0.382 \\
$P$ value & 0.000 & 0.253 & 0.138 & 0.107
\end{tabular}

ADC, apparent diffusion coefficient.

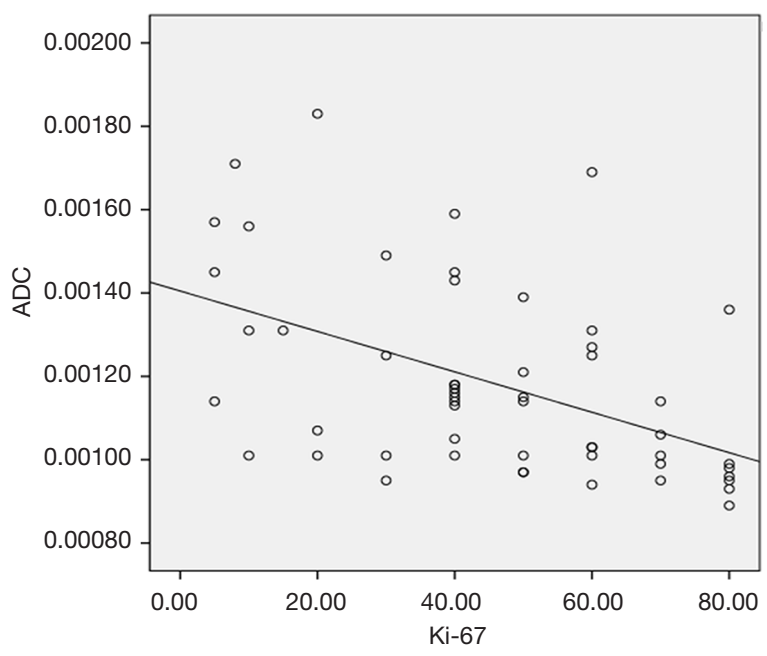

Figure 4 The average Ki-67 index of lung cancer was moderately correlated with $\mathrm{ADC}(\mathrm{r}=-0.506, \mathrm{P}=0.000<0.05)$. ADC, apparent diffusion coefficient.

pathological types of lung cancer. The count of tumor cells of squamous cell carcinoma and small cell lung cancer were higher than that of adenocarcinoma. At the same time, this study analyzed whether there was statistical difference in the expression level of $\mathrm{Ki}-67$ index among 3 different pathological types of lung cancer. Our results showed that there was a statistical difference in the expression level of Ki-67 index in patients with 3 different pathological types of lung cancer $(\mathrm{H}=103.762, \mathrm{P}=0.000)$. The results are similar to those reported by previous researchers (14). It also indicates that the participants in our study are suitable for further study of the correlation analysis between $\mathrm{Ki}-67$ index and image features. Previous studies have basically focused on correlation analysis between Ki-67 index and CT signs or enhancement features, which has detected a certain correlation between the Ki-67 index with CT signs or enhancement features $(15,16)$. However, there have been few studies on the comparison of Ki-67 index and MR signs.

In terms of comparing the correlation between $\mathrm{Ki}$ -
67 index and ADC value in different pathological types in lung cancer, our results showed that the average ADC value of adenocarcinoma was the highest, and it was lowest in small cell carcinoma. This study showed that there was a moderately negative correlation between average $\mathrm{ADC}$ value and Ki-67 index without distinguishing the pathological types. However, this correlation had also been confirmed in other solid tumors, such as breast cancer and glioma $(17,18)$. Therefore, this study indicates that ADC value can indirectly reflect the proliferation situation of Ki67 index in lung cancer. The higher the ADC value, the lower the cell proliferation activity.

By comparing the correlation between $\mathrm{Ki}-67$ index and $\mathrm{ADC}$ value in distinguishing pathological types of lung cancer, this study showed that there was no statistical difference. However, there have been few comparisons between different types of lung cancer and Ki-67 index in previous literatures based on MR examination. Most studies have been based on CT imaging features (19). In addition, the results of our study may be subject to the small number of participants and fewer cases of lung cancer targeted at the same stage. Therefore, further study should be carried out in a larger sample according to the same stage of lung cancer.

Compared to CT, MR examination has some advantages in evaluating the proliferation activity of lung cancer, such as the internal structure (solid and necrotic areas) of the tumor can be showed more clearly, the proliferative activity of the tumor is measured more accurately. In addition, it has certain advantages in showing the lymph nodes of hilum and mediastinum. However, there are some disadvantages of MR examination, such as long scanning time, high cost and small lesions of the lung cannot be showed clearly. Overall, there is more value in assessing the proliferative activity of lung cancer of MR examination.

This study had several limitations. First, it was a retrospective study in a single-center. Second, the sample size of patients who underwent MR examination was relatively small. Third, the measurement results may have 
been affected to a certain extent because of the lack of unified measurement standards (ROI size, location, etc.). Finally, our study was not based on the same staging of lung cancer.

\section{Conclusions}

As shown in our study, ADC value is expected to be the imaging indicators for predicting the expression level of Ki67 index in lung cancer, and as an important imaging basis for the prediction of invasion and prognosis in lung cancer.

\section{Acknowledgments}

Funding: This study was funded by grants from the Public Welfare Research Project of Jiaxing (No.: 2019AD32130) and 2019 Jiaxing Key Discipline of Medicine-Medical Imageology (Assisting Subject) (No.: 2019-fc-06).

\section{Footnote}

Reporting Checklist: The authors have completed the MDAR reporting checklist. Available at https://dx.doi. org/10.21037/tcr-21-2515

Data Sharing Statement: Available at https://dx.doi. org/10.21037/tcr-21-2515

Conflicts of Interest: All authors have completed the ICMJE uniform disclosure form (available at https://dx.doi. org/10.21037/tcr-21-2515). All authors have no conflicts of interest to declare.

Ethical Statement: The authors are accountable for all aspects of the work in ensuring that questions related to the accuracy or integrity of any part of the work are appropriately investigated and resolved. This study was approved by the Ethics Committee of the First Hospital of Jiaxing. The requirement for individual consent was waived by the committee due to retrospective nature of the study. All procedures performed in this study involving human participants were in accordance with the Declaration of Helsinki (as revised in 2013).

Open Access Statement: This is an Open Access article distributed in accordance with the Creative Commons Attribution-NonCommercial-NoDerivs 4.0 International License (CC BY-NC-ND 4.0), which permits the non- commercial replication and distribution of the article with the strict proviso that no changes or edits are made and the original work is properly cited (including links to both the formal publication through the relevant DOI and the license). See: https://creativecommons.org/licenses/by-nc-nd/4.0/.

\section{References}

1. Jemal A, Bray F, Center MM, et al. Global cancer statistics. CA Cancer J Clin 2011;61:69-90.

2. Wei DM, Chen WJ, Meng RM, et al. Augmented expression of $\mathrm{Ki}-67$ is correlated with clinicopathological characteristics and prognosis for lung cancer patients: an up-dated systematic review and meta-analysis with 108 studies and 14,732 patients. Respir Res 2018;19:150.

3. Wang D, Chen D, Zhang C, et al. Analysis of the relationship between Ki-67 expression and chemotherapy and prognosis in advanced non-small cell lung cancer. Transl Cancer Res 2020;9:3491-8.

4. Wen $\mathrm{S}, \mathrm{Zhou} \mathrm{W}, \mathrm{Li} \mathrm{CM}$, et al. Ki-67 as a prognostic marker in early-stage non-small cell lung cancer in Asian patients: a meta-analysis of published studies involving 32 studies. BMC Cancer 2015;15:520.

5. Peerlings J, Troost EG, Nelemans PJ, et al. The Diagnostic Value of MR Imaging in Determining the Lymph Node Status of Patients with Non-Small Cell Lung Cancer: A Meta-Analysis. Radiology 2016;281:86-98.

6. Li M, Wan Y, Zhang L, et al. Synchronous multiple lung cancers presenting as multifocal pure ground glass nodules: are whole-body positron emission tomography/computed tomography and brain enhanced magnetic resonance imaging necessary? Transl Lung Cancer Res 2019;8:649-57.

7. Mori T, Nomori H, Ikeda K, et al. Diffusion-weighted magnetic resonance imaging for diagnosing malignant pulmonary nodules/masses: comparison with positron emission tomography. J Thorac Oncol 2008;3:358-64.

8. Ohba Y, Nomori H, Mori T, et al. Is diffusion-weighted magnetic resonance imaging superior to positron emission tomography with fludeoxyglucose F 18 in imaging non-small cell lung cancer? J Thorac Cardiovasc Surg 2009;138:439-45.

9. Usuda K, Zhao XT, Sagawa M, et al. Diffusion-weighted imaging is superior to positron emission tomography in the detection and nodal assessment of lung cancers. Ann Thorac Surg 2011;91:1689-95.

10. Wu LM, Xu JR, Hua J, et al. Can diffusion-weighted 
imaging be used as a reliable sequence in the detection of malignant pulmonary nodules and masses? Magn Reson Imaging 2013;31:235-46.

11. Shen G, Hu S, Deng H, et al. Performance of DWI in the Nodal Characterization and Assessment of Lung Cancer: A Meta-Analysis. AJR Am J Roentgenol 2016;206:283-90.

12. Satoh S, Kitazume Y, Ohdama S, et al. Can malignant and benign pulmonary nodules be differentiated with diffusionweighted MRI? AJR Am J Roentgenol 2008;191:464-70.

13. Uto T, Takehara Y, Nakamura Y, et al. Higher sensitivity and specificity for diffusion-weighted imaging of malignant lung lesions without apparent diffusion coefficient quantification. Radiology 2009;252:247-54.

14. Huang YN, Zhao ZH, Mao HJ, et al. Correlation between DCE-MRI quantitative perfusion histogram parameters, apparent diffusion coefficient and Ki-67 in different pathological types of lung cancer. Zhonghua Yi Xue Za Zhi 2019;99:1645-50.

15. Chen C, Zhu WD, Zhang XH, et al. Value of Ki-67 and

Cite this article as: Ren $\mathrm{H}, \mathrm{Ma} \mathrm{J}$, Wang $\mathrm{J}$. Correlation between apparent diffusion coefficient and Ki-67 in different pathological types of lung cancer. Transl Cancer Res 2021;10(12):5364-5371. doi: 10.21037/tcr-21-2515 computed tomography in the assessment of peripheral lung adenocarcinoma. Br J Biomed Sci 2016;73:32-7.

16. Chen M, Li X, Wei Y, et al. Spectral CT imaging parameters and $\mathrm{Ki}-67$ labeling index in lung adenocarcinoma. Chin J Cancer Res 2020;32:96-104.

17. Martincich L, Deantoni V, Bertotto I, et al. Correlations between diffusion-weighted imaging and breast cancer biomarkers. Eur Radiol 2012;22:1519-28.

18. Yan R, Haopeng P, Xiaoyuan F, et al. Non-Gaussian diffusion MR imaging of glioma: comparisons of multiple diffusion parameters and correlation with histologic grade and MIB-1 (Ki-67 labeling) index. Neuroradiology 2016;58:121-32.

19. Zhou B, Xu J, Tian Y, et al. Correlation between radiomic features based on contrast-enhanced computed tomography images and $\mathrm{Ki}-67$ proliferation index in lung cancer: A preliminary study. Thorac Cancer 2018;9:1235-40. 